\title{
ATOMIC DATA REQUIREMENTS FOR THE ANALYSIS OF CHEMICALLY PECULIAR SPECTRA
}

\author{
D. S. LECKRONE \\ NASA/Goddard Space Flight Center, Greenbelt, MD 20771, USA \\ S. G. JOHANSSON, G. M. WAHLGREN AND T. BRAGE \\ Lund University, Lund Sweden \\ AND \\ C. R. PROFFITT \\ Computer Sciences Corporation and Catholic University of America
}

\section{Introduction}

For the past seven years a large, international team of astrophysicists and atomic physicists has been engaged in the systematic exploration and analysis of the extraordinary spectrum of one chemically-peculiar star, chi Lupi (B9.5pHgMn + A2 Vm). The observational data consist of 345 $\AA$ of the star's ultraviolet spectrum acquired with the Goddard High Resolution Spectrograph (GHRS) on board the Hubble Space Telescope (HST) at a resolving power of approximately 90,000 and an average $\mathrm{S} / \mathrm{N}$ ratio of about 75 in the continuum. These data were supplemented with new optical-wavelength echelle spectra obtained at the AAT and at KPNO[1]. We have named this study "The $\chi$ Lupi Pathfinder Project", because it is in many respects similar to the initial exploration of a strange new land - never before has the ultraviolet spectrum of an ultra-sharp-lined star been observed at this level of detail and accuracy.

We chose $\chi$ Lupi as the target for such intensive analysis because it is very bright $(V=3.9)$ and so could be efficiently observed $10 \AA$ at a time with the GHRS; it is extremely sharp-lined $\left(v \sin i=1, v_{\text {turb }}=0\right)$; and its abundance anomalies are among the most extreme, including large isotopic anomalies. The specific objectives of the $\chi$ Lupi Pathfinder Project are 1 . to explore and map at high resolution the UV-Optical spectrum of an ultra-sharp-lined CP star, 2. to derive LTE abundances that fully span the periodic table and investigate possible departures from LTE, 3 . to provide comprehensive constraints for state-of-the-art diffusion calculations, 4 . to provide a unique astrophysical "light source" for atomic spectroscopy, and 5. to provide a roadmap for the spectroscopy of other B- and A-type stars.

Spectrum synthesis and abundance analysis are initially based on Kurucz homogeneous, planeparallel model atmospheres for both the primary and secondary stellar components of this doublelined binary system, utilizing Kurucz's SYNTHE code and his extensive data base of atomic data derived from the Cowan Code. The beauty of working with ultraviolet and optical spectra simultaneously is that one has, in many cases, access to lines covering a wide range of excitation and multiple ionization states of a given element. Internal inconsistencies in abundances derived from lines covering such a range of conditions then provide clues about departures from the simple assumptions of LTE and atmospheric homogeneity. For example, ionization anomalies - descrepancies among abundances derived for different ionizations states of the same element - are a frequent occurrence in our analyses (e.g. Zr II versus Zr III). We have done an exhaustive state-of-the-art non-LTE analysis of $\mathrm{Hg} \mathrm{I}, \mathrm{Hg}$ II and $\mathrm{Hg}$ III, using the non-LTE code TLUSTY[2] and find only small departures from LTE in the ion fractions. From this we conclude that the rather large ionization anomalies seen in $\mathrm{Hg}$ in $\chi$ Lupi probably reflect an inhomogeneous radial distribution of $\mathrm{Hg}$ in the star's outer layers. 


\section{Atomic Data}

At a conference in Amsterdam in 1989, just prior to the launch of the HST/GHRS, we called attention to the very serious atomic data problems we would face in attempting to analyze GHRS spectra: "We face the bleak prospect of attempting to analyse spectra of $1 \%$ precision, obtained at great expense, with atomic parameters that can be inaccurate by factors of 2 or 10 , if they exist at all. The GHRS will be relentless in highlighting this problem." This prediction was immediately borne out. The then existing published database of energy levels, classified transitions, wavelengths, oscillator strengths, and nuclear effects (hyperfine structure and isotope shifts) was hopelessly inadequate in its coverage and accuracy to allow interpretation of the exquisitely detailed UV stellar spectrum we began to acquire in 1990. The existence of the GHRS observations of $\chi$ Lupi in fact has stimulated a resurgence of work around the world on the atomic spectra of the first several ionization states of many elements. $\chi$ Lupi is now widely cited by atomic physicists as a spectroscopic "standard light source". The synergy between atomic physics and astrophysics on this project has led to new insights in both fields. I can only briefly summarize the new atomic data that we are using. Further details may be found elsewhere[3,4].

The starting point for our analyses is Kurucz's massive semi-empirical data base, derived using the Cowan Code. It provides a unique and valuable source of wavelengths, oscillator strengths, and damping constants for millions of transitions, which are of highly variable accuracy (but even the less accurate data provide useful information about where further work is needed). We use Kurucz's data for general spectrum synthesis and line-blending calculations and to "fill in" the large number of transitions needed in non-LTE and diffusion calculations. For very accurate oscillator strengths, hyperfine structure constants and isotope shifts of specific, important transitions, we turn to the Multiconfiguration Dirac-Fock (MCDF) code developed by Charlotte Froese Fischer and adapted for our use by Tomas Brage[5]. This provides large-scale (many levels included), fully relativistic $a b$ initio calculations of atomic structure. It is often able to compute atomic data accurate to $10-20 \%$, and we use it for detailed abundance analyses and for the most important transitions in non-LTE and diffusion calculations. We also acquire very accurate oscillator strengths from level lifetimes measured (to $0.3 \mathrm{~ns}$ accuracy) in laser-induced fluorescense observations in the laboratory at the University of Lund[6] and at the University of Wisconsin[7], combined with branching ratios obtained from laboratory spectra. Under the best circumstances, we are able to measure the wavelengths of stellar features in the GHRS spectra to an accuracy of $0.5 \mathrm{~m} \AA$ - much more accurately than most published transition wavelengths. The Fourier Transform Spectrometers (FTS's) at the University of Lund and at Imperial College London provide wavelengths in the ultraviolet to accuracies of about $0.1 \mathrm{~m} \AA$, as well as the very high resolution required to measure hyperfine structure and isotope shifts[8]. Our colleagues at NIST[9] have provided important measurements of the wavelengths of lines of pure isotopes to accuracies of about $1 \mathrm{~m} \AA$ - critical to assessing some of the isotope abundance anomalies observed in $\chi$ Lupi.

On many occasions these superb, state-of-the-art new laboratory measurements or atomic calculations have "come to our rescue" in allowing us to interpret previously unexplained features in the $\chi$ Lupi spectrum. A good example is our discovery of resolved hyperfine lines of isotopically anomalous Tl II (ionized thallium, $Z=81$ ) - the first detection of this element in a star other than the $\operatorname{sun}[10,11,12]$.

\section{Abundance Survey Results}

At the start of our project abundances had been measured (from optical spectra) for approximately 10-12 elements and in most cases for only a single ionization state. Today we have derived abundances for 67 ions, encompassing 48 elements - and we have much more work to do. The current set of derived abundances for $\chi$ Lupi is plotted in Figure 1. A comprehensive discussion of these results may be found elsewhere[13], and an atlas of all the $\chi$ Lupi GHRS spectra is in preparation (to be submitted to AJ). Briefly summarized, the heavy element peak is completely defined by $\mathrm{Pt}, \mathrm{Au}, \mathrm{Hg}$ and $\mathrm{Tl}(Z=78-81)$, which are overabundant by $4-5$ orders of magnitude. Outside this peak, the most overabundant element is $\operatorname{Pd}(Z=46)$. The most underabundant elements are $\mathrm{Zn}$ (depleted by more than $4 \mathrm{dex}$ ), and B (we've positively detected the very weak B II $1362 \AA$ resonance line, which indicates boron is depleted by about $2.4 \mathrm{dex}$ ). Abundance patterns seen so far, appear qualitatively consistent with radiatively-driven diffusion - the most enhanced or depleted elements are 


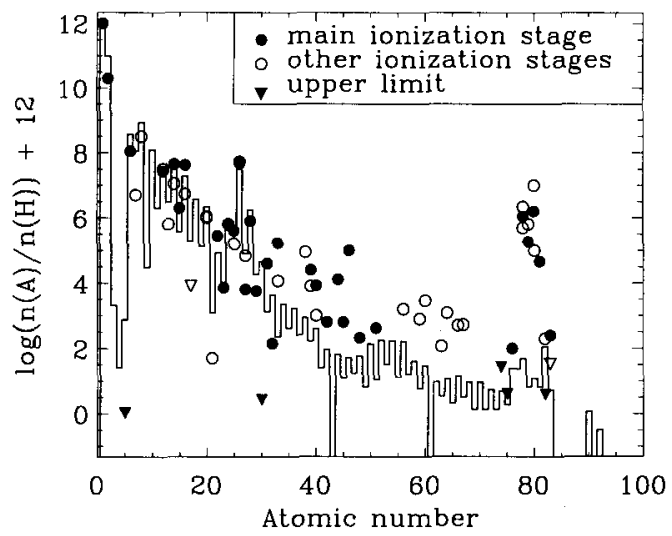

Figure 1. The abundances of chemical elements in $\chi$ Lupi (dots) are compared to those of the solar system (histogram). Filled circles mark abundances determined from the dominant ionization stage of that element, while open circles show those determined from other ionisation stages. Triangles denote upper limits.

species that were originally trace elements in the pre-stellar nebula (starting abundances less than 4-5 on the scale where hydrogen $=12$ ), and hence did not saturate the available radiation field. Species with higher starting abundances tend to be somewhat underabundant - presumably the dark cores of their strongest lines shielded them from the transfer of radiative momentum, so they tend to sink below the photosphere. We see plausible qualitative abundance trends along homolgous sequences (columns) of the periodic table. For example, the most enhanced element, $\mathrm{Hg}$, and the most depleted element, $\mathrm{Zn}$, are spectroscopically similar (two $s$ electrons outside a closed shell). So they should be roughly equally efficient in absorbing radiation. If $\mathrm{Hg}$ is very efficiently pushed upward into the photosphere, three-times-lighter $\mathrm{Zn}$ must be blown completely away from the star by radiation pressure - and this is qualitatively what we see. Quantitatively, however, radiation pressure is unable, in our sophisticated diffusion calculations, to sustain the enormous observed overabundances of such species as $\mathrm{Hg}$ and $\mathrm{Tl}$, at least in a homogeneous distribution through the photosphere. This is not an equilibrium situation. Theoretically, we would expect these elements to sink back to sub-photospheric regions. However, we need to investigate further whether an equilbrium condition can be found for an inhomogeneous radial abundance distribution, of the kind suggested by the large ionization anomalies derived for $\mathrm{Hg}$.

\section{References}

1. Wahlgren, G. M., Adelman, S. J., \& Robinson, R. D. (1994), ApJ, 434, 349.

2. Hubeny, I., \& Lanz, T. (1995), ApJ, 439, 875.

3. Leckrone, D. S., Johansson, S.G., Wahlgren, G. M., \& Adelman, S. J. (1993), Physica Scripta, T47, 149.

4. Leckrone, D. S., Johansson, S. G., Wahlgren, G. M., Proffitt, C. R., \& Brage, T. (1996), Physica Scripta, T65, 110.

5. Brage, T., Proffitt, C. R., \& Leckrone, D. S. (1998), ApJ, submitted.

6. Lundberg, H., Johansson, S. G., Larsson, J., Leckrone, D. S., Litzen, U., Svanberg, S., Wahlgren, G. M., \& Zerne, R. (1996), ApJ, 469, 388 .

7. Lawler, J. E., Bergeson, S. D., \& Wamsley, R.C. (1993), Physica Scripta, T47, 29.

8. Kalus, G., Johansson, S. G., Wahlgren, G. M., Leckrone, D.S., Thorne, A. P., \& Brandt, J. C. (1997), ApJ, in press.

9. Sansonetti, C. J., \& Reader, J. (1993), Phys. Rev. A, 47, 3080.

10. Leckrone, D. S., Johansson, S. G., Kalus, G., Wahlgren, G.M., Brage, T., \& Proffitt, C. R. (1996), ApJ, 462, 937.

11. Johansson, S.G., Kalus, G., Brage, T., Leckrone, D.S., \& Wahlgren, G.M. (1996), ApJ, 462, 943.

12. Brage, T., Leckrone, D. S., \& Froese Fischer, C. (1996), Phys. Rev. A, 53, 192.

13. Leckrone, D. S., Johansson, S. G., Wahlgren, G. M., Proffitt, C. R., \& Brage, T. (1997), in "The Scientific Impact of the Goddard High Resolution Spectrograph," ed. J. C. Brandt, T. B. Ake, \& C. C. Petersen, ASP Conf. Ser., in press, p. 135. 